OPEN ACCESS

Edited by:

Rocío Martínez De Pablos,

Universidad de Sevilla, Spain

Reviewed by:

Gloria Patricia Cardona Gomez, Universidad de Antioquía, Colombia Alla B. Salmina,

Krasnoyarsk State Medical University named after Prof.

V.F.Voino-Yasenetski, Russia

${ }^{*}$ Correspondence: Kaoru Sato

kasato@nihs.go.jp

Received: 04 July 2018 Accepted: 30 November 2018 Published: 13 December 2018

Citation:

Shigemoto-Mogami Y, Hoshikawa K and Sato K (2018) Activated Microglia

Disrupt the Blood-Brain Barrier and Induce Chemokines and Cytokines in

a Rat in vitro Model.

Front. Cell. Neurosci. 12:494.

doi: 10.3389/fncel.2018.00494

\section{Activated Microglia Disrupt the Blood-Brain Barrier and Induce Chemokines and Cytokines in a Rat in vitro Model}

\author{
Yukari Shigemoto-Mogami ${ }^{1,2}$, Kazue Hoshikawa ${ }^{1}$ and Kaoru Sato ${ }^{1 *}$ \\ ${ }^{1}$ Laboratory of Neuropharmacology, Division of Pharmacology, National Institute of Health Sciences, Kawasaki-ku, Japan, \\ ${ }^{2}$ Department of Neuropharmacology, Interdisciplinary Graduate School of Medicine, University of Yamanashi, Yamanashi, \\ Japan
}

Severe neuroinflammation is associated with blood brain barrier (BBB) disruption in CNS diseases. Although microglial activation and the subsequent changes in cytokine/chemokine (C/C) concentrations are thought to be key steps in the development of neuroinflammation, little data are available concerning the interaction of microglia with BBB cells. In this study, we investigated this interaction by adding LPS-activated microglia (LPS-MG) to the abluminal side of a BBB model composed of endothelial cells (EC), pericytes (Peri) and astrocytes (Ast). We then examined the abluminal concentrations of $27 \mathrm{C} / \mathrm{C}$ s and the interactions between the LPS-MG and BBB cells. LPS-MG caused collapse of the BBB, revealed by decreases in the trans-endothelial electrical resistance (TEER) and by changes in the expression levels of tight junction (TJ) proteins. Under these conditions, $19 \mathrm{C} / \mathrm{Cs}$ were markedly increased on the abluminal side. Unexpectedly, although LPS-MG alone released 10 of the $19 \mathrm{C} / \mathrm{Cs}$, their concentrations were much lower than those detected on the abluminal side of the BBB model supplemented with LPS-MG. Co-culture of LPS-MG with Ast caused marked increases in 12 of the $19 \mathrm{C} / \mathrm{Cs}$, while co-culture of LPS-MG with EC and Peri resulted in a significant increase in only 1 of the $19 \mathrm{C} / \mathrm{Cs}$ (fractalkine). These results suggest that $\mathrm{C} / \mathrm{C}$ dynamics in this system are not only caused by activated microglia but also are due to the interaction between activated microglia and astrocytes.

Keywords: BBB disruption, microglia, inflammation, cytokine, chemokine

\section{INTRODUCTION}

The blood brain barrier (BBB) acts as a protective barrier of the central nervous system (CNS) against potential neurotoxic molecules (Abbott et al., 2010; Serlin et al., 2015). BBB functions are regulated by various types of cells that belong to a neurovascular unit (NVU) (Zlokovic, 2011; Keaney and Campbell, 2015). Recent reports have clarified that microglia not only act as the CNS-resident immune cells but also have important physiological roles in the CNS (ShigemotoMogami et al., 2014; Sato, 2015). Microglia sense slight changes in the surrounding environment and associate along the brain capillaries (Nimmerjahn et al., 2005). In a variety of pathological conditions (Oby and Janigro, 2006; Desai et al., 2007; McCaffrey et al., 2008; Bataveljic et al., 2014; Ortiz et al., 2014; Kamphuis et al., 2015; Li et al., 2015; van de Haar et al., 2016), functional 
impairment of BBB has been reported (Zhao et al., 2015; Almutairi et al., 2016) and is often correlated with disease severity. Microglia adopt an activated form in pathological conditions in which BBB breakdown is a hallmark (Nakajima and Kohsaka, 1993; Kreutzberg, 1996; Graeber and Streit, 2010; Salter and Beggs, 2014; Streit et al., 2014). Furthermore, activated microglia produce inflammatory cytokines such as TNF $\alpha$ and IL-1 $\beta$ (Nishioku et al., 2010; Yang et al., 2015) that increase $\mathrm{BBB}$ permeability and downregulate TJ proteins ( $\mathrm{Gu}$ et al., 2015; Almutairi et al., 2016). However, little is known about the comprehensive changes in cytokines/chemokines $(\mathrm{C} / \mathrm{Cs})$ and the interaction between microglia and the other NVU cells in neuroinflammation associated with $\mathrm{BBB}$ collapse. In this study, we measured the concentrations of $27 \mathrm{C} / \mathrm{Cs}$ when $\mathrm{BBB}$ collapse was induced by activated microglia in an in vitro BBB model (Nakagawa and Niwa, 2009; Nakagawa et al., 2009). We found that the interactions of activated microglia with pericytes and endothelial cells, and with astrocytes were critical in determining the final concentrations of $\mathrm{C} / \mathrm{Cs}$.

\section{MATERIALS AND METHODS}

This study was carried out in accordance with the principles of the Basel Declaration and recommendations of Guide for the Care and Use of Laboratory Animals, the Animal Research Committee of the National Institute of Health Sciences, Japan. The protocol was approved by the Animal Research Committee of the National Institute of Health Sciences, Japan.

\section{Materials}

Bovine serum albumin (BSA), Evans blue, sodium fluorescein $(\mathrm{NaF})$ and anti- $\beta$-actin antibody (A5316) were purchased from Sigma-Aldrich (St. Louis MO, United States). Fetal bovine serum (FBS) and Dulbecco's Modified Eagle's Medium (DMEM) were purchased from Life Technologies (Grand Island, NY, United States). The rat in vitro model of the BBB (RBT-24H) was purchased from PharmaCo-Cell Company Ltd. (Nagasaki, Japan). Anti-ZO-1 (33-9100), anti-claudin5 (35-2500), and antioccludin (33-1500) antibodies were purchased from Invitrogen (Camarillo CA, United States). Anti-GRO KC (AF-515), antiGFAP (AF-2594) antibodies were purchased from R\&D systems (Minneapolis, MN, United States). Anti-Iba1 (019-19741) antibody and DAPI were purchased from Wako (Osaka, Japan). The MILLIPLEX MAP Rat Cytokine/Chemokine Panel was purchased from Merck Millipore (Billerica MA, United States). SuperSignal West Femto Substrate was purchased from Thermo Scientific (Rockford IL, United States). Can Get Signal was purchased from TOYOBO (Osaka, Japan).

\section{Preparation of the Rat in vitro BBB Model}

The in vitro $\mathrm{BBB}$ model was cultured according to the manufacturer's protocol. Microglia were added to the abluminal side and incubated for 1 day (Figure 1A).

\section{Microglial Cell Culture}

Rat microglia were cultured as previously described (Nakajima et al., 1992; Nakajima and Kohsaka, 1993; Shigemoto-Mogami et al., 2014). To activate microglia, they were incubated with $1 \mu \mathrm{g} / \mathrm{ml}$ LPS for $1 \mathrm{~h}$ (LPS-MG), which has already been shown to induce inflammatory reaction in our preliminary experiments and another's report (Huang et al., 2018). Microglia were then washed twice, and transferred to the abluminal side of the BBB model at $5.0 \times 10^{4}$ cells $/ \mathrm{cm}^{2}$.

\section{Measurement of the Transendothelial Electrical Resistance (TEER)}

The TEER was measured by an Endohm resistance meter (World Precision Instruments, FL. United States).

\section{Measurement of Transcellular Transport and Paracellular Transport}

To measure transcellular transport and paracellular transport, we measured the permeabilities of Evans blue albumin (EBA) and $\mathrm{NaF}$, respectively. EBA $(165 \mu \mathrm{g} / \mathrm{ml})$ and $\mathrm{NaF}(10 \mu \mathrm{g} / \mathrm{ml})$ were added to the luminal side. PBS-H (10 mM HEPES, $25 \mathrm{mM}$ glucose in PBS) was added to the transmembranes $(30 \mathrm{~min})$ and the concentrations of $\mathrm{NaF}$ and EBA in the PBS-H were measured using a Spectra Max (Molecular Devices, CA. United States). The permeability coefficient $\left(\mathrm{P}_{\text {app }}\right)$ was calculated using the following formula: $\mathrm{P}_{\mathrm{app}}(\mathrm{cm} / \mathrm{s})=\mathrm{V}_{\mathrm{A}} / \mathrm{A} \times[\mathrm{C}]_{\text {luminal }} \mathrm{x} \Delta[\mathrm{C}]_{\text {Abluminal }} / \Delta \mathrm{t}$.

\section{Western Blotting of TJ Proteins}

The transmembranes with EC and Peri were lysed with sample buffer (62.5 mM Tris, 2\% SDS, $10 \%$ glycerin, $0.0125 \%$ bromophenol blue, $\mathrm{pH}$ 6.8), and homogenized on ice. The concentrations of primary antibodies: anti-ZO-1 [1:1,000], anticlaudin5 [1:2,000], anti-occludin [1:1,000], anti- $\beta$-actin [1:5,000]. We have confirmed that the bands were detected with their specific molecular weights, i.e., ZO-1(225 kDa), claudin5 (24 $\mathrm{kDa})$, occludin $(65 \mathrm{kDa})$, and $\beta$-actin $(42 \mathrm{kDa})$. The level of $\beta$-actin was not changed in any experimental conditions.

\section{Immunocytochemistry}

The expressions of ZO-1 and claudin5 in EC and Peri, and the expression of GRO $\mathrm{KC}$ in co-culture of Ast and MG were examined immunocytochemically (Shigemoto-Mogami et al., 2014). The primary antibodies were used in the following concentrations: anti-ZO-1 [1:100], anti-claudin5 [1:100], antiGRO KC [1:100], anti-GFAP [1:50], anti-Iba1 [1:100]. For visualization of nuclei, DAPI was used $[1: 1,000]$.

\section{Measurement of $\mathrm{C} / \mathrm{Cs}$}

The concentrations of 27 rat C/Cs (IL- $1 \alpha$, IL-1 $\beta$, IL-2, IL-4, IL5, IL-6, IL-10, IL-12 (p70), IL-13, IL-17, IL-18, EGF, eotaxin, fractalkine, G-CSF, GM-CSF, GRO/KC, IFN- $\gamma$, IP-10, leptin, LIX, MCP-1, MIP-1 $\alpha$, MIP-2, RANTES, TNF $\alpha$, and VEGF) were measured by MILLIPLEX MAP Multiplex Immunoassay Kits (Rat Cytokine/Chemokine Magnetic bead kit RCYPMX27MAG).

\section{Data Analysis and Statistics}

The statistical analysis was performed using ANOVA followed by Tukey's test. Differences were considered significant at a value 
A

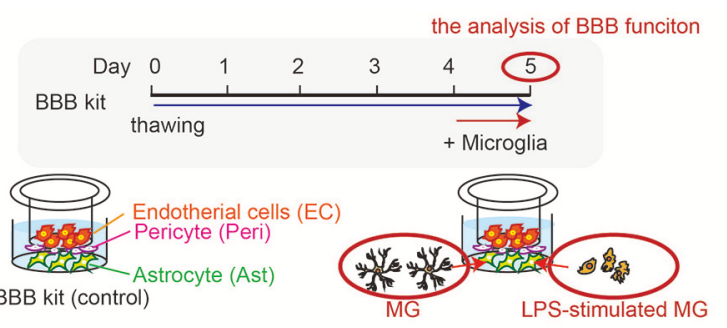

B

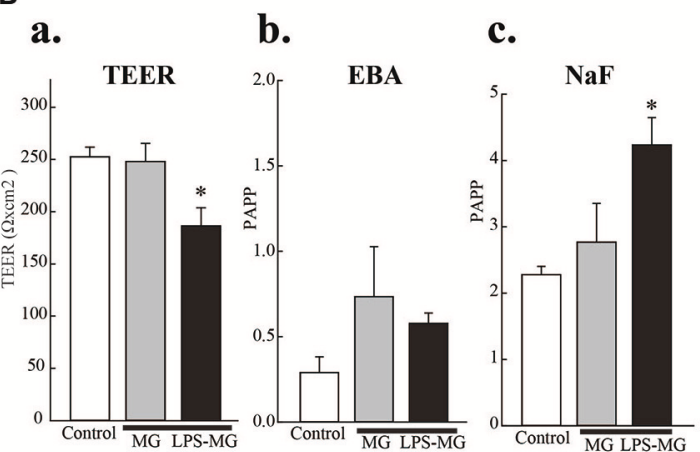

d.
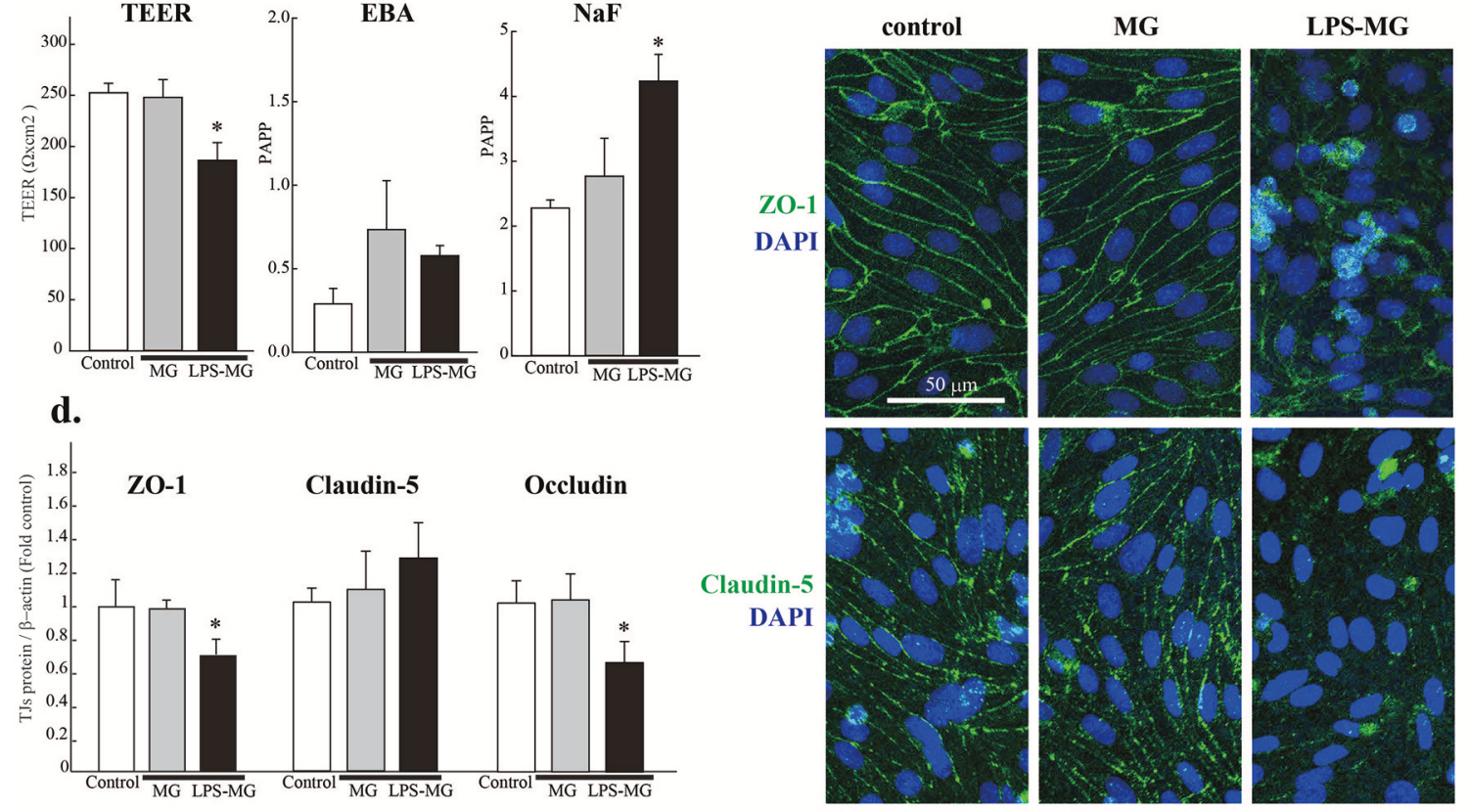

e.

C

a.

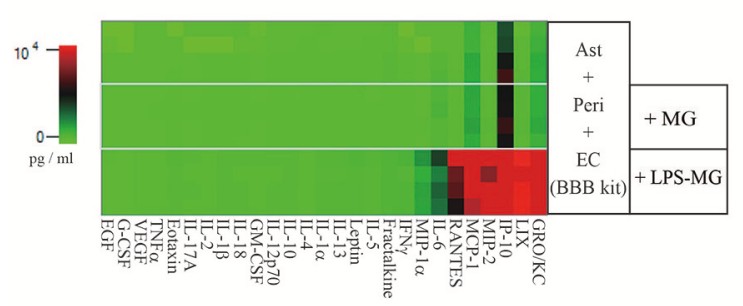

b.

\begin{tabular}{|c|c|c|c|c|}
\hline & & $+\mathrm{MG}$ & +LPS-MG & \multirow{2}{*}{$\begin{array}{l}\text { +LPS-MG } \\
\text { / BBB kit }\end{array}$} \\
\hline & \multicolumn{3}{|c|}{ Ast + Peri + EC (BBB kit) } & \\
\hline GRO KC & $264.3 \pm 4.7$ & $255.8 \pm 23.7$ & $13486.0 \pm 1580.2 *$ & 51.0 \\
\hline LIX & $22.3 \pm 2.4$ & $27.0 \pm 3.8$ & $9048.0 \pm 0.0^{*}$ & 405.6 \\
\hline IP-10 & $1352.5 \pm 154.7$ & $1314.5 \pm 70.7$ & $3521.2 \pm 426.2^{*}$ & 2.6 \\
\hline MIP-2 & $6.5 \pm 5.3$ & $18.4 \pm 2.8$ & $3088.0 \pm 884.1 *$ & 469.3 \\
\hline MCP-1 & $288.0 \pm 1.9$ & $300.2 \pm 14.4$ & $2992.5 \pm 567.9^{*}$ & 10.4 \\
\hline RANTES & $1.6 \pm 1.3$ & $6.7 \pm 2.2$ & $1830.0 \pm 288.2^{*}$ & 1143.8 \\
\hline $\mathrm{IL}-6$ & $63.3 \pm 6.8$ & $82.2 \pm 22.1$ & $703.7 \pm 90.4 *$ & 11.1 \\
\hline MIP-1 $\alpha$ & $0.6 \pm 0.5$ & $2.6 \pm 0.5$ & $328.9 \pm 41.7 *$ & 506.1 \\
\hline $\mathrm{IFN} \gamma$ & $16.8 \pm 3.6$ & $10.0 \pm 4.1$ & $92.1 \pm 13.5^{*}$ & 5.5 \\
\hline Fractalkine & $27.8 \pm 6.1$ & $12.9 \pm 0.8$ & $81.9 \pm 7.1^{*}$ & 2.9 \\
\hline IL-5 & $17.7 \pm 0.9$ & $20.9 \pm 0.9$ & $60.9 \pm 2.9^{*}$ & 3.4 \\
\hline leptin & $16.0 \pm 0.8$ & $13.4 \pm 1.2$ & $54.6 \pm 4.9^{*}$ & 3.4 \\
\hline $\mathrm{IL}-13$ & $9.1 \pm 0.9$ & $10.2 \pm 2.2$ & $45.7 \pm 4.6^{*}$ & 5.0 \\
\hline $\mathrm{IL}-1 \alpha$ & ND & $2.4 \pm 2.8$ & $42.3 \pm 7.5^{*}$ & ND \\
\hline $\mathrm{IL}-4$ & $4.3 \pm 0.8$ & $5.1 \pm 0.3$ & $32.2 \pm 3.3^{*}$ & 7.4 \\
\hline IL-10 & $5.4 \pm 0.2$ & $4.9 \pm 0.9$ & $31.0 \pm 3.9^{*}$ & 5.7 \\
\hline $\mathrm{LL}-12 \mathrm{p} 70$ & $9.6 \pm 0.0$ & $9.9 \pm 1.1$ & $30.3 \pm 2.2^{*}$ & 3.2 \\
\hline GM-CSF & $3.0 \pm 2.0$ & $1.7 \pm 2.0$ & $29.8 \pm 5.8$ & 9.8 \\
\hline IL-18 & $7.1 \pm 4.7$ & $8.3 \pm 1.0$ & $29.7 \pm 4.1$ & 4.2 \\
\hline $\mathrm{LL}-1 \beta$ & $1.7 \pm 0.1$ & $1.4 \pm 0.6$ & $24.3 \pm 3.4^{*}$ & 13.8 \\
\hline $\mathrm{IL}-2$ & ND & ND & $22.6 \pm 5.8$ & ND \\
\hline $\mathrm{IL}-17 \mathrm{~A}$ & ND & ND & $19.8 \pm 2.0$ & ND \\
\hline Eotaxin & $4.2 \pm 0.8$ & $4.5 \pm 0.9$ & $15.7 \pm 1.6$ & 3.7 \\
\hline TNF $\alpha$ & ND & ND & $15.4 \pm 1.4^{*}$ & ND \\
\hline VEGF & $8.5 \pm 0.6$ & $11.8 \pm 2.0$ & $11.6 \pm 1.2$ & 1.4 \\
\hline G-CSF & ND & ND & $2.3 \pm 1.7$ & ND \\
\hline EGF & ND & ND & $0.3 \pm 0.0$ & ND \\
\hline
\end{tabular}

FIGURE 1 | Activated microglia disrupt BBB barrier functions and cause concentration changes of $19 \mathrm{C} / \mathrm{Cs}$. (A) Schematic diagram of the experiment. (B) Effects of LPS-MG on TEER (a), and the permeability of EBA (b) and NaF (c). Effects of LPS-MG on the expression levels of TJ proteins (d). Immunocytochemistry of ZO-1 (top) (Continued) 
FIGURE 1 | and claudin5 (bottom) (e). Scale bar indicates $50 \mu \mathrm{m} . \mathrm{N}=4,{ }^{*}<0.05$ vs. control, ANOVA followed by Tukey's test. Error bars represent the s.e.m. (C) Comprehensive quantitative measurement of C/C concentrations in the medium of the abluminal side of the BBB model one day after incubation with LPS-MG. (a) Heat map of the concentrations of all $27 \mathrm{C} / \mathrm{Cs}$. (b) Concentrations and their fold changes. The fold change represents the C/C concentration ratio of LPS-MG + BBB to BBB alone. Asterisks indicate a significant increase compared to the BBB alone. $N=4,{ }^{*}<0.05$ vs. control, ANOVA followed by Tukey's test. The reproducibility of the data was confirmed by 3 independent experiments.
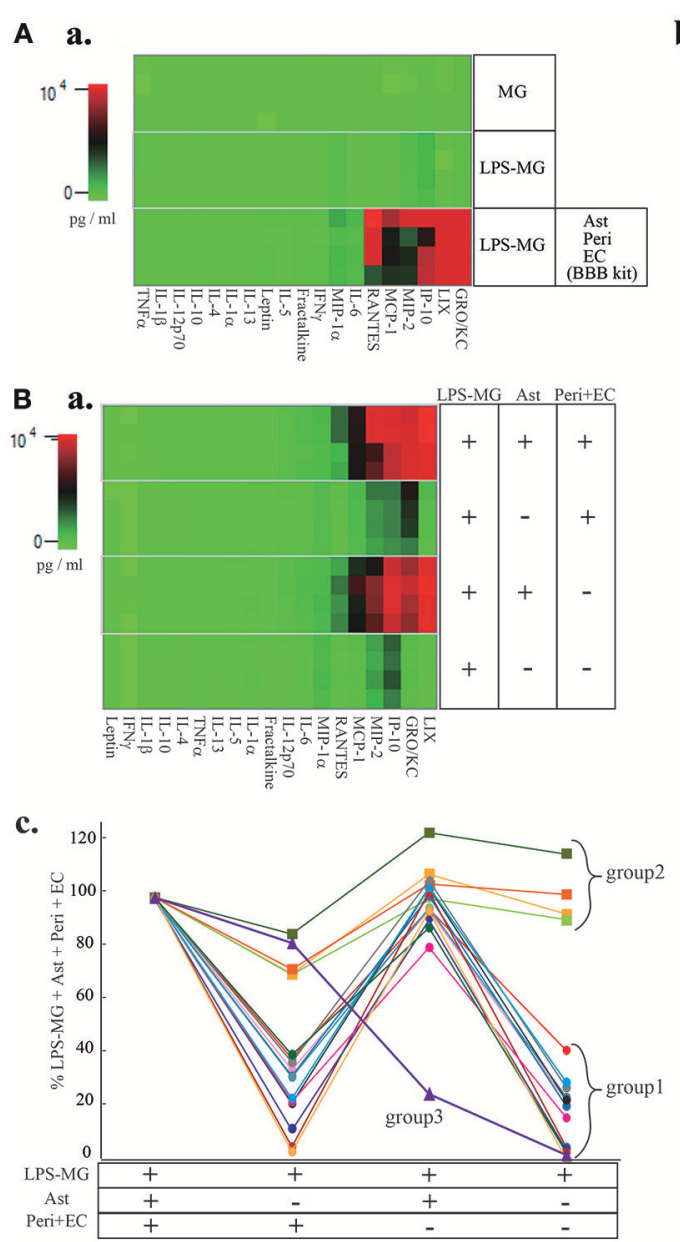

b.

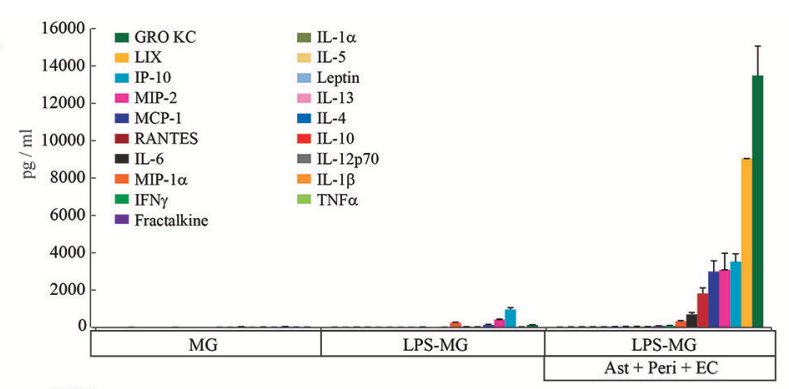

b.

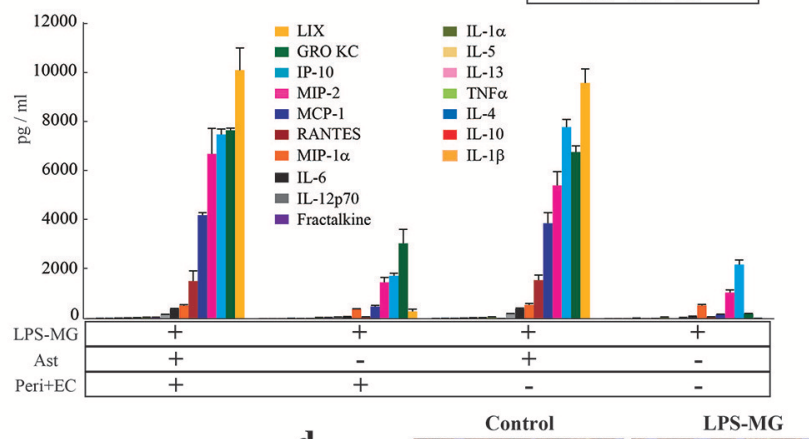

d.

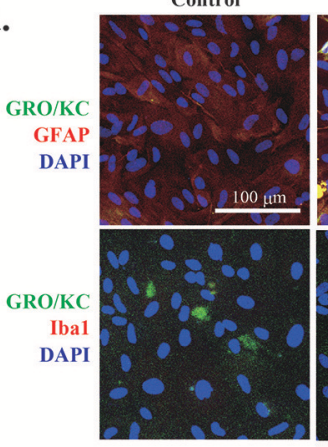

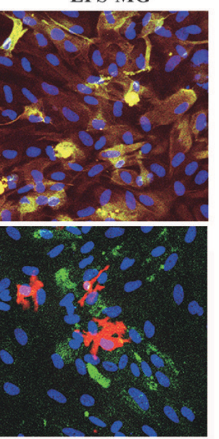

FIGURE 2 | The interaction of LPS-MG with the other NVU cells is important for C/C dynamics. (A) After 1 day of incubation with LPS-MG, the concentrations of all $27 \mathrm{C} / \mathrm{Cs}$ were measured. (a) The heat map of the $27 \mathrm{C} / \mathrm{Cs}$ in microglia alone, LPS-MG alone and the LPS-MG + BBB model. (b) Comparison of the concentrations of the $19 \mathrm{C} / \mathrm{Cs}$ upregulated by LPS-MG (see Fig. 1) in the above three culture conditions. (B) The $19 \mathrm{C} / \mathrm{C}$ concentrations in the LPS-MG + BBB model, the LPS-MG + Peri + EC, LPS-MG + Ast, and LPS-MG. (a) Heat map of the $19 \mathrm{C} / \mathrm{C}$ concentrations in the above four culture conditions. (b,c) Concentrations of the C/Cs in all the experimental conditions: (b) raw concentrations; (c) those normalized to the LPS-MG + BBB model. The $19 \mathrm{C} / \mathrm{Cs}$ were divided into three groups. (d) Typical expression pattern of cell type specific markers (GFAP, Iba1) (red) and that of GRO KO (green) in the co-culture of LPS-MG and astrocytes were shown. Scale bar indicates $100 \mu \mathrm{m}$. The reproducibility of the data was confirmed by 3 independent experiments.

of $p<0.05$. The reproducibility of the data was confirmed by 3 independent experiments.

\section{RESULTS}

We first attempted to reproduce the BBB disruption observed in neuroinflammation by adding LPS-MG to the abluminal side of the BBB model (Figures 1A,Ba-e). A 1 day incubation with LPSMG significantly decreased the TEER and increased paracellular transport $(\mathrm{NaF})$. In addition, the expression levels of occludin and ZO-1 were significantly decreased (Figure 1Bd). As revealed by immunostaining of $\mathrm{ZO} 1$ and claudin5, TJ structures were collapsed by LPS-MG (Figure 1Be). When we added microglia without LPS stimulation, no changes were detected in these parameters. We then measured the concentrations of $27 \mathrm{C} / \mathrm{Cs}$ on the abluminal side of the BBB model. The basal levels of $\mathrm{C} / \mathrm{Cs}$, without microglia, were identical to levels after adding unstimulated microglia concentrations (shown in a heat map, Figure $\mathbf{1 C a}$ and quantitatively, Figure $\mathbf{1} \mathbf{C b}$ ). However, adding LPS-MG significantly increased the concentrations of $19 \mathrm{C} / \mathrm{Cs}$ 
(GRO/KC, LIX, IP-10, MIP-2, MCP-1, RANTES, IL-6, MIP-1 $\alpha$, IFN- $\gamma$, fractalkine, IL-5, leptin, IL-13, IL-1 $\alpha$, IL-4, IL-10, IL12 (p70), IL-1 $\beta$, and TNF $\alpha$ ). The right column of Figure $\mathbf{1 C b}$ indicates the ratios of C/Cs with LPS-MG to those at basal levels, i.e., the induction strength.

We next attempted to identify the cell types responsible for the changes in $\mathrm{C} / \mathrm{Cs}$ (Figure 2). We first measured $\mathrm{C} / \mathrm{C}$ concentrations in supernatants of microglia alone, of LPS-MG alone, and of the medium on the abluminal side of the BBB model supplemented with LPS-MG (Figure 2Aa,b). Microglia alone did not affect the concentrations of C/Cs, but LPS-MG increased $10 \mathrm{C} / \mathrm{Cs}$ (IL-1 $\beta$, IL-10, IL-12 (p70), GRO/KC, IP-10, MCP-1, MIP-1 $\alpha$, RANTES, MIP-2, and TNF $\alpha$ ). However, the concentrations of these $\mathrm{C} / \mathrm{Cs}$ were much lower than $\mathrm{C} / \mathrm{Cs}$ of the BBB model supplemented with LPS-MG. These results suggest that the interaction of LPS-MG with BBB cells is important in determining the final concentrations of $\mathrm{C} / \mathrm{Cs}$. To determine which of the $\mathrm{BBB}$ components increased the $\mathrm{C} / \mathrm{C}$ concentrations, we compared the $\mathrm{C} / \mathrm{C}$ concentrations in the entire LPS-MG + BBB model to that of LPS-MG co-cultured only with Peri + EC, co-cultured only with Ast, and LPS-MG cells alone, without any $\mathrm{BBB}$ components (Figure 2B). The heat map of $\mathrm{C} / \mathrm{Cs}$ in the LPS-MG + Ast and LPS-MG + BBB conditions were very similar (Figure $\mathbf{2} \mathbf{B a}$ ). Figure $\mathbf{2} \mathbf{B b}$ and $\mathrm{c}$ show the concentrations of the respective $\mathrm{C} / \mathrm{Cs}$ in the four culture conditions. The 19 $\mathrm{C} / \mathrm{Cs}$ appeared to be divided into three groups. Group 1 (LIX, GRO/KC, IP-10, MIP-2, MCP-1, RANTES, IL-6, IL-12 (p70), IL-5, IL-13, IL-4, and IL-10) included C/Cs of which the final concentrations were determined by the interaction between LPSMG and Ast. When we performed double immunostaining of GRO KC, one of the members of this group, along with cell type specific markers, we found GRO KC associated with astrocytes after the stimulation with LPS-MG (Figure 2Bd, top), but not with microglia (bottom). Group 2 (MIP-1 $\alpha$, IL- $1 \alpha$, TNF $\alpha$, and IL$1 \beta$ ) included $\mathrm{C} / \mathrm{Cs}$ of which the final concentrations depended on LPS-MG. Group 3 included fractalkine; the concentration was mainly determined by the Peri + EC condition, and Ast suppressed its release.

\section{DISCUSSION}

The addition of activated microglia to the abluminal side of the BBB model caused a collapse of the BBB. For example, the membrane-specific localization of $\mathrm{ZO}-1$ and claudin 5 almost disappeared after microglial addition. Activated microglia also induced drastic changes in the concentrations of $\mathrm{C} / \mathrm{Cs}$, indicating that microglial activation can trigger $\mathrm{BBB}$ disruption during neuroinflammation. Among $19 \mathrm{C} / \mathrm{Cs}$ that showed significant changes in our experiment, IP-10 (Chai et al., 2015), MCP-1 (Yao and Tsirka, 2014), IL-6 (Paul et al., 2003), IL-1 $\beta$ (Argaw et al., 2006; Wang et al., 2014), and TNF $\alpha$ (Afonso et al., 2007) have previously been reported to induce $\mathrm{BBB}$ disruption. The concentrations of GRO/KC (Maysami et al., 2015), LIX (Wang et al., 2016), MIP-2 (Shaftel et al., 2007), RANTES (Ubogu et al., 2006), and MIP-1 $\alpha$ (Maysami et al., 2015) are reported to be elevated at the time of $\mathrm{BBB}$ collapse, which causes infiltration of monocytes and $\mathrm{T}$ cells into the CNS, thereby worsening $\mathrm{BBB}$ disruption. The $\mathrm{C} / \mathrm{Cs}$ described above may act as both a cause and a consequence of the neuroinflammation.

Many studies have suggested that activated microglia themselves disrupt $\mathrm{BBB}$ integrity by releasing inflammatory $\mathrm{C} / \mathrm{Cs}$ (Yenari et al., 2006; da Fonseca et al., 2014). However, we found that although activated microglia alone induced significant elevations of GRO/KC, IP-10, MIP-2, MCP-1, RANTES, MIP- $1 \alpha$, IL-12 (p70), IL-10, IL-1 $\beta$, TNF $\alpha$, and IL- $1 \alpha$, their concentration changes were slight. The concentrations of $12 \mathrm{C} / \mathrm{Cs}$ (LIX, GRO/KC, IP-10, MIP-2, MCP-1, RANTES, IL-6, IL-12 (p70), IL-5, IL-13, IL-4, and IL-10) were mainly determined by the interaction between astrocytes and LPS-MG. The 7 most highly concentrated C/Cs (GRO KC, LIX, IP-10, MIP-2, MCP-1, RANTES, and IL-6) in Figure $\mathbf{1 B b}$ were included in this group. LIX (Wang et al., 2016), GRO/KC (Maysami et al., 2015), IP-10 (Chai et al., 2015), MIP-2 (Shaftel et al., 2007), MCP-1 (Yao and Tsirka, 2014), RANTES (Ubogu et al., 2006), IL-6 (Paul et al., 2003), and IL-10 (Lin et al., 2018) play roles in BBB disruption during neuroinflammation. By immunostaining, we confirmed that the main resource of GRO KC was astrocytes, while only minor signals were detected in LPS-MG. Further experiments are necessary to clarify the contribution of astrocytes and activated microglia to the final concentration of each cytokine. The concentrations of IL- $1 \beta$, TNF $\alpha$, IL- $1 \alpha$, and MIP- $1 \alpha$ in the above 4 culture conditions were the same as those with LPS-MG alone, indicating that activated microglia are the predominant source of these C/Cs. However, their concentrations were lower than those in the co-culture of LPS-MG and Ast. These four C/Cs are wellknown pro-inflammatory C/Cs (Wang et al., 2014; Rochfort et al., 2016) and might trigger the subsequent serial $\mathrm{C} / \mathrm{C}$ concentration changes leading to BBB collapse. The concentration change of fractalkine was unique. The main source of fractalkine was Peri + EC, i.e., blood vessels, and microglia suppressed the fractalkine release.

In this study, activated microglia-induced $\mathrm{C} / \mathrm{Cs}$ were categorized into three groups based on the main sources, i.e., microglia, Ast, and EC + Peri (blood vessels). Of note, microglia-triggered elevation of Ast $\mathrm{C} / \mathrm{Cs}$ was remarkable in terms of both quantity and variation. BBB collapse and the subsequent infiltration of peripheral immune cells into brain parenchyma drastically worsen neuroinflammation (Dos Passos et al., 2016; Zenaro et al., 2017). It therefore seems likely that signals between microglia and Ast are potent therapeutic targets for neuroinflammation.

\section{AUTHOR CONTRIBUTIONS}

KS: designed research; YS-M, KH, and KS: performed research; YS-M, KH, and KS: analyzed data; YS-M and KS: wrote the paper.

\section{FUNDING}

Grant from AMED for project focused on developing key evaluation technology: development of platform technology for drug discovery through application of regenerative medicine (17be0304302j0101, 18be0304207j0102) Grant-in-Aid for Scientific Research (C) from MEXT (17K08330). 


\section{REFERENCES}

Abbott, N. J., Patabendige, A. A., Dolman, D. E., Yusof, S. R., and Begley, D. J. (2010). Structure and function of the blood-brain barrier. Neurobiol. Dis. 37, 13-25. doi: 10.1016/j.nbd.2009.07.030

Afonso, P. V., Ozden, S., Prevost, M. C., Schmitt, C., Seilhean, D., Weksler, B., et al. (2007). Human blood-brain barrier disruption by retroviral-infected lymphocytes: role of myosin light chain kinase in endothelial tight-junction disorganization. J. Immunol. 179, 2576-2583. doi: 10.4049/jimmunol.179.4.2576

Almutairi, M. M., Gong, C., Xu, Y. G., Chang, Y., and Shi, H. (2016). Factors controlling permeability of the blood-brain barrier. Cell. Mol. Life Sci. 73, 57-77. doi: 10.1007/s00018-015-2050-8

Argaw, A. T., Zhang, Y., Snyder, B. J., Zhao, M. L., Kopp, N., Lee, S. C., et al. (2006). IL-1beta regulates blood-brain barrier permeability via reactivation of the hypoxia-angiogenesis program. J. Immunol. 177, 5574-5584. doi: 10.4049/jimmunol.177.8.5574

Bataveljic, D., Milosevic, M., Radenovic, L., and Andjus, P. (2014). Novel molecular biomarkers at the blood-brain barrier in ALS. BioMed Res. Int. 2014:907545. doi: 10.1155/2014/907545

Chai, Q., She, R., Huang, Y., and Fu, Z. F. (2015). Expression of neuronal CXCL10 induced by rabies virus infection initiates infiltration of inflammatory cells, production of chemokines and cytokines, and enhancement of blood-brain barrier permeability. J. Virol. 89, 870-876. doi: 10.1128/JVI.02154-14

da Fonseca, A. C., Matias, D., Garcia, C., Amaral, R., Geraldo, L. H., Freitas, C., et al. (2014). The impact of microglial activation on blood-brain barrier in brain diseases. Front. Cell. Neurosci. 8:362. doi: 10.3389/fncel.2014.00362

Desai, B. S., Monahan, A. J., Carvey, P. M., and Hendey, B. (2007). Blood-brain barrier pathology in Alzheimer's and Parkinson's disease: implications for drug therapy. Cell Transl. 16, 285-299. doi: 10.3727/000000007783464731

Dos Passos, G. R., Sato, D. K., Becker, J., and Fujihara, K. (2016). Th17 cells pathways in multiple sclerosis and neuromyelitis optica spectrum disorders: pathophysiological and therapeutic implications. Mediators Inflamm. 2016:5314541. doi: 10.1155/2016/5314541

Graeber, M. B., and Streit, W. J. (2010). Microglia: biology and pathology. Acta Neuropathol. 119, 89-105. doi: 10.1007/s00401-009-0622-0

Gu, X., Wei, Z. Z., Espinera, A., Lee, J. H., Ji, X., Wei, L., et al. (2015). Pharmacologically induced hypothermia attenuates traumatic brain injury in neonatal rats. Exp. Neurol. 267, 135-142. doi: 10.1016/j.expneurol.2015.02.029

Huang, M. Y., Tu, C. E., Wang, S. C., Hung, Y. L., Su, C. C., Fang, S. H., et al. (2018). Corylin inhibits LPS-induced inflammatory response and attenuates the activation of NLRP3 inflammasome in microglia. BMC Complement. Alter. Med. 18:221. doi: 10.1186/s12906-018-2287-5

Kamphuis, W. W., Derada Troletti, C., Reijerkerk, A., Romero, I. A., and de Vries, H. E. (2015). The blood-brain barrier in multiple sclerosis: microRNAs as key regulators. CNS Neurol. Disord. Drug Targets 14, 157-167. doi: $10.2174 / 1871527314666150116125246$

Keaney, J., and Campbell, M. (2015). The dynamic blood-brain barrier. FEBS J. 282, 4067-4079. doi: 10.1111/febs.13412

Kreutzberg, G. W. (1996). Microglia: a sensor for pathological events in the CNS. Trends Neurosci. 19, 312-318. doi: 10.1016/0166-2236(96)10049-7

Li, F., Wang, Y., Yu, L., Cao, S., Wang, K., Yuan, J., et al. (2015). Viral infection of the central nervous system and neuroinflammation precede blood-brain barrier disruption during japanese encephalitis virus infection. J. Virol. 89, 5602-5614. doi: 10.1128/JVI.00143-15

Lin, R., Chen, F., Wen, S., Teng, T., Pan, Y., and Huang, H. (2018). Interleukin-10 attenuates impairment of the blood-brain barrier in a severe acute pancreatitis rat model. J. Inflamm. 15:4. doi: 10.1186/s12950-018-0180-0

Maysami, S., Haley, M. J., Gorenkova, N., Krishnan, S., McColl, B. W., and Lawrence, C. B. (2015). Prolonged diet-induced obesity in mice modifies the inflammatory response and leads to worse outcome after stroke. J. Neuroinflamm. 12:140. doi: 10.1186/s12974-015-0359-8

McCaffrey, G., Seelbach, M. J., Staatz, W. D., Nametz, N., Quigley, C., Campos, C. R., et al. (2008). Occludin oligomeric assembly at tight junctions of the blood-brain barrier is disrupted by peripheral inflammatory hyperalgesia. $J$. Neurochem. 106, 2395-2409. doi: 10.1111/j.1471-4159.2008.05582.x

Nakagawa, S., Deli, M. A., Kawaguchi, H., Shimizudani, T., Shimono, T., Kittel, A., et al. (2009). A new blood-brain barrier model using primary rat brain endothelial cells, pericytes and astrocytes. Neurochem. Int. 54, 253-263. doi: 10.1016/j.neuint.2008.12.002

Nakagawa, S., and Niwa, M. (2009). Blood-brain barrier (BBB). Nihon yakurigaku zasshi Folia pharmacologica Japonica 133, 170-172.

Nakajima, K., and Kohsaka, S. (1993). Functional roles of microglia in the brain. Neurosci. Res. 17, 187-203. doi: 10.1016/0168-0102(93)90047-T

Nakajima, K., Tsuzaki, N., Shimojo, M., Hamanoue, M., and Kohsaka, S. (1992). Microglia isolated from rat brain secrete a urokinase-type plasminogen activator. Brain Res. 577, 285-292. doi: 10.1016/0006-8993(92)90285-H

Nimmerjahn, A., Kirchhoff, F., and Helmchen, F. (2005). Resting microglial cells are highly dynamic surveillants of brain parenchyma in vivo. Science 308, 1314-1318. doi: 10.1126/science.1110647

Nishioku, T., Matsumoto, J., Dohgu, S., Sumi, N., Miyao, K., Takata, F., et al. (2010). Tumor necrosis factor-alpha mediates the blood-brain barrier dysfunction induced by activated microglia in mouse brain microvascular endothelial cells. J. Pharmacol. Sci. 112, 251-254. doi: 10.1254/jphs.09292SC

Oby, E., and Janigro, D. (2006). The blood-brain barrier and epilepsy. Epilepsia 47, 1761-1774. doi: 10.1111/j.1528-1167.2006.00817.x

Ortiz, G. G., Pacheco-Moises, F. P., Macias-Islas, M. A., Flores-Alvarado, L. J., Mireles-Ramirez, M. A., Gonzalez-Renovato, E. D., et al. (2014). Role of the blood-brain barrier in multiple sclerosis. Arch. Med. Res. 45, 687-697. doi: 10.1016/j.arcmed.2014.11.013

Paul, R., Koedel, U., Winkler, F., Kieseier, B. C., Fontana, A., Kopf, M., et al. (2003). Lack of IL-6 augments inflammatory response but decreases vascular permeability in bacterial meningitis. Brain 126, 1873-1882. doi: 10.1093/brain/awg171

Rochfort, K. D., Collins, L. E., McLoughlin, A., and Cummins, P. M. (2016). Tumour necrosis factor-alpha-mediated disruption of cerebrovascular endothelial barrier integrity in vitro involves the production of proinflammatory interleukin-6. J. Neurochem. 136, 564-572. doi: 10.1111/jnc.13408

Salter, M. W., and Beggs, S. (2014). Sublime microglia: expanding roles for the guardians of the CNS. Cell 158, 15-24. doi: 10.1016/j.cell.2014.06.008

Sato, K. (2015). Effects of microglia on neurogenesis. Glia 63, 1394-1405 doi: $10.1002 /$ glia. 22858

Serlin, Y., Shelef, I., Knyazer, B., and Friedman, A. (2015). Anatomy and physiology of the blood-brain barrier. Semi. Cell Dev. Biol. 38, 2-6. doi: 10.1016/j.semcdb.2015.01.002

Shaftel, S. S., Carlson, T. J., Olschowka, J. A., Kyrkanides, S., Matousek, S. B., and O’Banion, M. K. (2007). Chronic interleukin-1beta expression in mouse brain leads to leukocyte infiltration and neutrophil-independent blood brain barrier permeability without overt neurodegeneration. J. Neurosci. 27, 9301-9309. doi: 10.1523/JNEUROSCI.1418-07.2007

Shigemoto-Mogami, Y., Hoshikawa, K., Goldman, J. E., Sekino, Y., and Sato, K. (2014). Microglia enhance neurogenesis and oligodendrogenesis in the early postnatal subventricular zone. J. Neurosci. 34, 2231-2243. doi: 10.1523/JNEUROSCI.1619-13.2014

Streit, W. J., Xue, Q. S., Tischer, J., and Bechmann, I. (2014). Microglial pathology. Acta Neuropathol. Commun. 2:142. doi: 10.1186/s40478-014-0142-6

Ubogu, E. E., Callahan, M. K., Tucky, B. H., and Ransohoff, R. M. (2006) Determinants of CCL5-driven mononuclear cell migration across the bloodbrain barrier. Implications for therapeutically modulating neuroinflammation. J. Euroimmunol. 179, 132-144. doi: 10.1016/j.jneuroim.2006. 06.004

van de Haar, H. J., Burgmans, S., Jansen, J. F., van Osch, M. J., van Buchem, M. A., Muller, M., et al. (2016). Blood-brain barrier leakage in patients with early alzheimer disease. Radiology 281, 527-535. doi: 10.1148/radiol.2016152244

Wang, L. Y., Tu, Y. F., Lin, Y. C., and Huang, C. C. (2016). CXCL5 signaling is a shared pathway of neuroinflammation and blood-brain barrier injury contributing to white matter injury in the immature brain. J. Neuroinflamm. 13:6. doi: 10.1186/s12974-015-0474-6

Wang, Y., Jin, S., Sonobe, Y., Cheng, Y., Horiuchi, H., Parajuli, B., et al. (2014). Interleukin-1beta induces blood-brain barrier disruption by downregulating Sonic hedgehog in astrocytes. PLoS ONE 9:e110024. doi: 10.1371/journal.pone.0110024

Yang, Y., Salayandia, V. M., Thompson, J. F., Yang, L. Y., and Estrada, E. Y. (2015). Attenuation of acute stroke injury in rat brain by minocycline promotes blood-brain barrier remodeling and alternative microglia/macrophage 
activation during recovery. J. Neuroinflamm. 12:26. doi: 10.1186/s12974-0150245-4

Yao, Y., and Tsirka, S. E. (2014). Monocyte chemoattractant protein1 and the blood-brain barrier. Cell. Mol. Life Sci. 71, 683-697. doi: $10.1007 / \mathrm{s} 00018-013-1459-1$

Yenari, M. A., Xu, L., Tang, X. N., Qiao, Y., and Giffard, R. G. (2006). Microglia potentiate damage to blood-brain barrier constituents: improvement by minocycline in vivo and in vitro. Stroke 37, 1087-1093. doi: 10.1161/01.STR.0000206281.77178.ac

Zenaro, E., Piacentino, G., and Constantin, G. (2017). The blood-brain barrier in Alzheimer's disease. Neurobiol. Dis. 107, 41-56. doi: 10.1016/j.nbd.2016. 07.007

Zhao, Z., Nelson, A. R., Betsholtz, C., and Zlokovic, B. V. (2015). Establishment and dysfunction of the blood-brain barrier. Cell 163, 1064-1078. doi: 10.1016/j.cell.2015.10.067
Zlokovic, B. V. (2011). Neurovascular pathways to neurodegeneration in Alzheimer's disease and other disorders. Nat. Rev. Neurosci. 12, 723-738. doi: $10.1038 / \mathrm{nrn} 3114$

Conflict of Interest Statement: The authors declare that the research was conducted in the absence of any commercial or financial relationships that could be construed as a potential conflict of interest.

Copyright (c) 2018 Shigemoto-Mogami, Hoshikawa and Sato. This is an open-access article distributed under the terms of the Creative Commons Attribution License (CC $B Y)$. The use, distribution or reproduction in other forums is permitted, provided the original author(s) and the copyright owner(s) are credited and that the original publication in this journal is cited, in accordance with accepted academic practice. No use, distribution or reproduction is permitted which does not comply with these terms. 